mammals. Psychological Review, 1966, 73, 409-426.

GRAY, P. H. Theory and evidence of imprinting in human infants. Joumal of Psychology, $1958,46,155-166$

HARPER, L. V. The effects of isolation from birth on the social behaviour of guinea pigs in adulthood. Animal Behaviour, 1968, 16 58-64.

HESS, E. H. Imprinting. Science, 1959, 130, 133-141.

SHIPLEY, W. U. The demonstration in the domestic guinea pig of a process resembling classical imprinting. Animal Behaviour, 1963, $11,470-474$.
SLUCKIN, W. Imprinting in guinea pigs. Nature, $1968,220,1148$

SLUCKIN, W., \& FULLERTON, C. Attachments of infant guinea pigs. Psychonomic Science, $1969,17,179-180$.

TOBACH, E., \& GOLD, P. S. Behavior of the guinea pig in the open-field situation. Psychological Reports, 1966, 18, 415-425.

\section{NOTE}

1. This research was conducted by the authors at Princeton University in 1966 and was supported in part by NIMH Grant M-1562 to B. A. Campbell.

\title{
Work rate with noncontingent supplement of the reward ${ }^{1}$
}

PETER R. HARLEY, University of Oregon, Eugene, Oreg. 97403, and ALFRED E. ETAUGH, Bradley University, Peoria, Ill. 61606

Rats were trained to work for food on a VI 1-min schedule. During the operant sessions, a buzzer sounded throughout intervals in which free pellets were delivered every 30 sec. Neither the appearance nor continuation of this superimposed condition altered established rates. However, upon buzzer-free-food termination, rates were depressed and became less regular.

Intuitively, it seems that the rate of a simple operant response would be subject to change with variations in the animal's general emotional state. Estes \& Skinner (1941) made a convincing case for this notion in experiments where they presented a tone for several minutes during the stable emission of an operant and then terminated the signal with a foot shock to the rat. Ultimately, the rat stopped responding in the presence of the tone and resumed only after the shock. The flat spot in the cumulative record is now known as a conditioned emotional response, or the effect of conditioned suppression (Hunt \& Brady, 1951). The signal may be thought of as a conditioned stimulus (CS), and the unavoidable shock, as an unconditioned stimulus (US).

Looking for a complement to the conditioned-suppression paradigm, we substituted food for foot shock in the above procedure. Brady (1961) had seen rate facilitation during a signal for upcoming intracranial reward, but Azrin \& Hake (1969) have - reported establishing suppression during a CS followed by water, food, or rewarding intracranial stimulation. Since we were unable to observe either suppression or facilitation when we delivered a single food pellet after a 30 -sec audible buzz to an animal working for these pellets, we deviated somewhat from the usual approach in hope of strengthening the effect of the CS. In these explorations, a serendipitous observation has suggested itself as a matter of general psychological interest.

\section{METHOD}

Four rats were kept at $80 \%$ of their ad lib weights in Cohen constant-weight cages for 2 weeks prior to their first experimental sessions. During these 2 weeks, all animals were habituated to the Skinner box and magazine trained, while two of the rats ( 3 and 6 ) were given 15 Pavlovian sessions. Pavlovian treatments were separated by as little as $45 \mathrm{~min}$ and as much as 3 days. In these treatments, all four rats were in their closely apposed home cages and a buzzer was sounded for $5 \mathrm{~min}$. Rats 3 and 6 were automatically presented a $97-\mathrm{mg}$ pellet every $30 \mathrm{sec}$ while the other two rats ( 2 and 4 ) received no food. At the end of these 2 weeks, all rats were given daily hour-long periods in a Skinner box programmed to food-reward with a $45-\mathrm{mg}$ pellet on a variable-interval (VI) 1-min schedule. Two- to 8-min buzzer presentations, in which a free pellet was delivered every $30 \mathrm{sec}$, were superimposed on each operant session of Rat 6, which had had food in its home-cage buzz, and on those of Rat 2, which had had no food in its home-cage buzz. "Signaled free gratification" (SFG) appears to be the least problematic general label for the condition, since the actual contingencies for food presentation are never altered. Buzzer presentations in which no free pellets were presented, and where the VI 1-min schedule was also unaltered, were superimposed on the schedules of the other two rats ( 1 and 3 ). Rats 2 and 6 were given 17 daily sessions prior to extinction, and Rats 1 and 3 were given 9 such periods prior to extinction.

\section{RESULTS AND DISCUSSION}

No demonstrable rate facilitation was produced in any of these efforts, and neither was there any suppressive effect caused by the onset and continuation of the buzzer or the buzzer and free food. On the fourth day, however, Rat 2 began to show a mild post-SFG depression, which continued to appear in subsequent sessions. In the seventh running of Rat 2, an equipment failure resulted in the uninterrupted continuation of the buzzer-free-food condition for the last $35 \mathrm{~min}$ of the session. Figure 1 shows that introducing the SFG had no effect on response rate, in spite of the fact that pellet delivery during the buzzer was $300 \%$ of the nonbuzzer value. But in the eighth session, the termination of SFG in its usual brief amount resulted in a marked decrement in responding. Subsequent buzzer-free-food superimpositions had the same effect; i.e., onset and continuation of SFG did not alter the existing rate, but its termination caused the rat to slow down appreciably. In addition, responding after the post-SFG depression became less regular than usual. Reintroducing the buzzer-free-food during post-SFG depression restored the previous rate immediately.

In the ninth session for Rat 6, we allowed SFG to persist for approximately the last $35 \mathrm{~min}$, and again there was no rate change during this situation. In the 10th and 11 th sessions, Rat 6 failed to show the post-SFG depression produced by Rat 2 . Therefore, in its 12th session, SFG was again superimposed on the final $35 \mathrm{~min}$. There were no rate changes in the 13th period, but in the 14 th, the post-SFG depression appeared and was evident thereafter. However, both depression and subsequent rate irregularities for this animal were less pronounced than they were for Rat 2.

During extinction, as one might expect (Estes, 1943, 1948), the buzzer appeared to facilitate responding by Rats 2 and 6 , and did not affect Rats 3 and 4 .

We have not yet studied the acquisition of an operant in the continued presence of SFG, nor have we looked at a rat's 


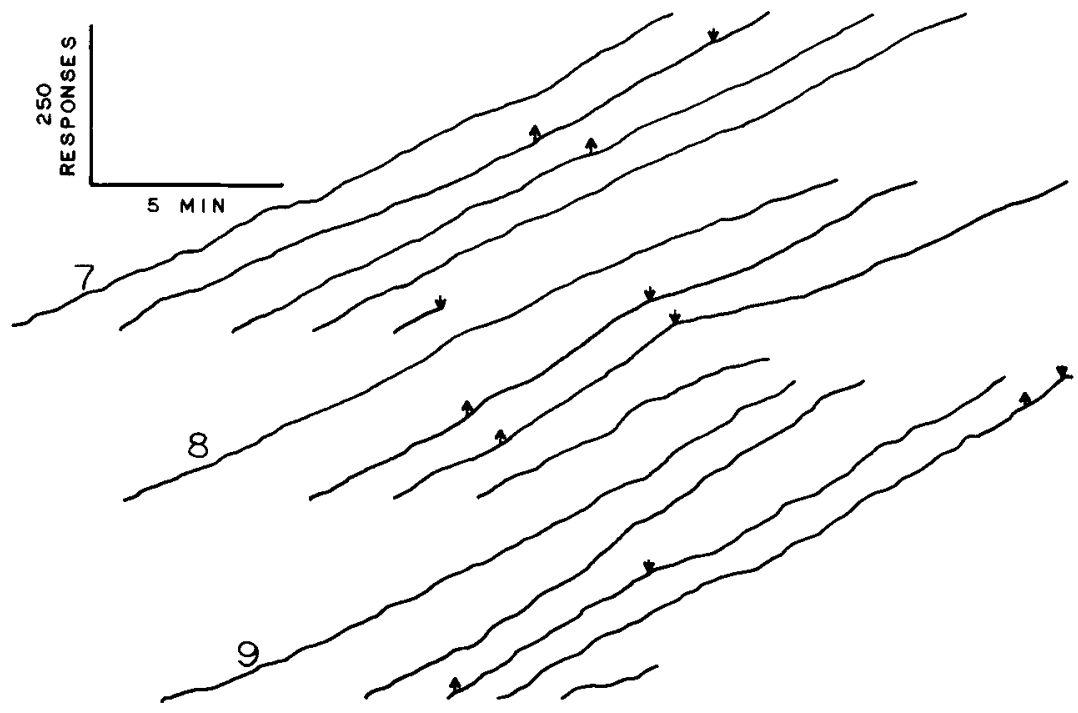

preference regarding work opportunities and pure SFG. Speculation about these prospects is withheld, for prior to the present report, it might have been most reasonably predicted (Skinner, 1948) that manipulations such as ours would lead to a rat's being adventitiously conditioned to misconstrue its daily sessions as multiple VI VI schedules. These are arrangements where it is indicated to the animal that it is working for one of a number of possible reinforcement densities. This in itself is not an acceptable interpretation of our results, since, in multiple schedules, a signaled change in reinforcement density is reflected in rate, and the component schedules are usually independent of one another (Ferster \& Skinner, 1957). However, it may be possible to characterize our results in terms of interactions between components of schedules (Reynolds, 1961 ), and this may ultimately prove the most satisfactory language for experiments such as these.

But our rats did not react as though their reinforcement density had been altered during the buzzer, and their response rate after SFG was not independent of its occurrence. The rats seem to have appreciated the introduction of SFG, and their only reaction is that, after having had it for some time, it upsets them to have it taken away.
Fig. 1. Sessions 7, 8, and 9 for Rat 2 . Arrows pointed up indicate buzzer-free-food on; down, off.

\section{REFERENCES}

AZRIN, N. H., \& HAKE, D. F. Positive conditioned suppression: Conditioned suppression using positive reinforcers as the unconditioned stimuli. Journal of the Experimental Analysis of Behavior, 1969, 12, $167-173$.

BRADY, J. V. Motivational-emotional factors and intracranial self-stimulation. In D. E. Sheer (Ed.), Electrical stimulation of the brain. Austin: University of Texas Press, 1961. Pp. 413-430.

ESTES, W. K. Discriminative conditioning: I. A discriminative property of conditioned anticipation. Journal of Experimental Psychology, 1943, 32, 150-155.

ESTES, W. K. Discriminative condition: II. Effects of a Pavlovian conditioned stimulus upon a subsequently established operant response. Journal of Experimental Psy chology, 1948, 32, 173-177.

ESTES, W. K., \& SKINNER, B' F. Some quantitative properties of anxiety. Journal of Experimental Psychology, 1941, 29, 390-400.

FERSTER, C. B., \& SKINNER, B. F. Schedules of reinforcement. New York: Appleton-Century, 1957.

HUNT, H. F., \& BRADY, J. V. Some effects of electroconvulsive shock on a conditioned emotional response ("anxiety"). Journal of Comparative \& Physiological Psychology, $1951,44,88-98$.

REYNOLDS, G. S. An analysis of interactions in a multiple schedule. Journal of the Experimental Analysis of Behavior, 1961, 4 , 107-117.

SKINNER, B. F. Superstition in the pigeon. Journal of Experimental Psychology, 1948, $38,168-172$.

\section{NOTE}

1. This work was done as the first author's Master's thesis while he was a student at Bradley University, 1966-67. 